How to reference this article

Barsotti, A. (2019). Ripensare a Cani di bancata di Emma Dante. I travestimenti androgini di Mammasantissima. Italica Wratislaviensia, 10(2), 289-305.

DOI: http://dx.doi.org/10.15804/IW.2019.10.1.30

\author{
Anna Barsotti \\ Università degli Studi di Pisa \\ anna.barsotti@unipi.it \\ ORCID: 0000-0001-6108-0524
}

\title{
RIPENSARE A CANI DI BANCATA DI EMMA DANTE. I TRAVESTIMENTI ANDROGINI DI MAMMASANTISSIMA
}

\author{
THINKING BACK TO CANI DI BANCATA \\ BY EMMA DANTE. MAMMASANTISSIMA'S \\ ANDROGYNOUS COSTUMES
}

\begin{abstract}
The essay reflects on Emma Dante, the unusual and versatile artist (theatre manager, actress-author, film and opera director). Emma Dante's story condenses different and apparently conflicting experiences and knowledge (the Academy of Dramatic Arts in Rome, the teachings of Vacis in Turin, the laboratories with Cesare Ronconi) before the foundation of the Sud Costa Occidentale group in Palermo, in 1999, with its subsequent transformations continuing until today. The result is an irregular figure of a "matriarch", in the fruitful vein of new Sicilian dramaturgy, which takes nourishment from the land of origin but with which she feeds a collective and authorial theatre. This theatre is both dramatic (indeed tragi-comic) and post-dramatic, with European depth, and it is not spared of controversy and criticism, as it is awkward and uncomfortable. The analysis of her performance in Cani di bancata (2006) aims to highlight themes and styles connected to a feminism that goes beyond gender in the strict sense but that is able to become a metaphor of a world and a human diversity that involves and disturbs us through an irreverent gaze.
\end{abstract}

Keywords: Emma Dante, theatre, Cani di bancata, Sicily, world 


\section{CORNICE}

E mma Dante è artista polivalente (capocomica e regista di teatro, attrice e regista di cinema, regista di opere liriche), ma costituisce un'eccezione nel panorama italiano in quanto "donna di scena" divenuta, ormai, anche "donna di libro". La sua formazione, all'inizio attorica, condensa esperienze e competenze diverse, perfino apparentemente contrastanti: Accademia d'Arte drammatica a Roma, magistero di Gabriele Vacis a Torino, laboratorio con Cesare Ronconi. Ne nasce un'anomala figura di "matriarca", nel filone fecondo della nuova drammaturgia siciliana, che dalla terra d'origine trae succhi ma con i quali alimenta un teatro al tempo stesso collettivo e autoriale; drammatico (anzi tragi-comico) e postdrammatico, di rispondenza europea, non senza suscitare polemiche e attacchi, specialmente nel suo paese, essendo teatro scomodo e foriero di rischiose "autoanalisi".

Ripensando a Cani di bancata (2006) $)^{3}$, il suo unico spettacolo sulla Mafia, dove "il" Mammasantissima è una donna, si può tentare di met-

1 La Dante ha esitato molto a diventare donna di libro: dopo avere pubblicato due testi in rivista nel 2003 (mPalermu, Carnezzeria) e nel 2007 (Cani di bancata), soltanto alla fine dello stesso anno ha dato alle stampe la Trilogia della famiglia siciliana, che comprende anche Vita mia (Dante, 2007b). Per approfondimenti rimando ai miei precedenti studi sull'autrice: Barsotti 2009, 2010, 2011, 2014, 2017.

2 «Il mio teatro è matriarcale», riconosce lei stessa (Ficara, Pompei, 2007, pp. 189$-196)$.

3 Cani di bancata, drammaturgia e regia: Emma Dante, assistente alla regia: Elisa Di Liberato, assistente alla drammaturgia: Eleonora Lombardo, scene: Emma Dante e Carmine Maringola, costumi: Emma Dante, disegno luci: Cristian Zucaro, foto: Giuseppe Distefano. Con: Manuela Lo Sicco (Mammasantissima), Antonio Puccia (Totò Siciliano detto “Zù Totò 'u bisturi’), Salvatore D’Onofrio (Salvatore Spagnuolo detto "Don Sasà"), Sandro Maria Campagna (Toni Cintola detto "Big Jim"), Carmine Maringola (Girolamo Riccio detto "Gegè 'u farmacista"), Sabino Civilleri (Stefano Varvarà detto "Slim Fast”), Michele Riondino/Alessandro Rognone (Gennaro Panzanella detto "Joker"), Alessio Piazza (Giuseppe Bonanno detto "Spatuzza"), Fabrizio Lombardo/Enzo Di Michele (Vito Montalto detto "Cicciobello"/“Topo Gigio”), Ugo Giacomazzi (Federico Panunzio detto "U purtiere"), Stefano Miglio (Cst Liborio Paglino). Produzione: Crt Centro di Ricerca per il Teatro di Milano in collaborazione con Palermo Teatro Festival. Presentato in anteprima al Nuovo Teatro Montevergini di 
tere in luce temi e stili connessi a un femminile che esula dal gender (in senso stretto) ma capace di farne la matrice o la metafora di una diversità umana che, per la sua ambivalenza, ci coinvolge e (per)turba. Lo spettacolo nasce, d'altronde, da una lunga gestazione processuale (Dalisi, 2007, pp. 108-109), e segna l'avvio di una nuova stagione nell'itinerario italiano ed europeo dell'artista siciliana ${ }^{4}$. Implica, per primo, l'ampliamento del gruppo originario Sud Costa Occidentale, formato nel 1999 a Palermo e composto da attori-coautori come Sabino Civilleri, Manuela Lo Sicco, Italia Carroccio, e Gaetano Bruno. Attraversato lo Stretto (e anche i confini della nazione) la capogruppo ha aperto le prove e moltiplicati laboratori in diverse città italiane, per consolidare un metodo "maieutico" capace di formare altri attori, in vista (o meno) di successive produzioni teatrali.

Lo spettacolo in esame scaturisce da tali operazioni e processi; a tutti gli effetti un testo-ponte. L'ampliamento non è soltanto numerico, frutto di un parziale ricambio generazionale e dell'innesto combinatorio con altre "terre di teatro", dove la lingua è corpo. Quella partenopea specialmente, per l'ingresso fra $i$ "cani di bancata" dei napoletani Carmine Maringola, che diventerà collaboratore e marito di Emma, e Salvatore D’Onofrio. Il tema stesso della Mafia necessita d'un ampliamento spa-

Palermo il 10 novembre 2006, debutta il 14 novembre dello stesso anno al Crt-Teatro dell'Arte di Milano. Ho visto lo spettacolo dal vivo a Cascina, alla Città del Teatro, il 20 gennaio 2007, e rivisto grazie alla ripresa video, effettuata in quella circostanza, dai tecnici dell'Università di Pisa. Per il testo edito di Cani di bancata faccio riferimento a Dante, 2007a, pp. 102-107; utile anche il video Emma Dante - Il gesto necessario, regia: Clarissa Cappellani, montaggio: Miss Silence, montaggio del suono: Eugenio Cerocchi, durata 47 minuti, 2007, Italia.

4 «Ci saranno poi altri spettacoli importanti fino a Le sorelle Macaluso, Premio Ubu 2015, la pubblicazione dei testi (dalle riviste specializzate alla Rizzoli), l'approdo alla Scala di Milano con Carmen, due premi alla Biennale di Venezia col film tratto dal suo romanzo, Via Castellana Bandiera (2013)» (Mariani, 2016, p. 113). Tra gli spettacoli più recenti si segnalano La Scortecata (2017) e Bestie di scena (2018). Emerge la ricorrenza o il rilievo di personaggi femminili nelle altre produzioni originali, come nelle regie liriche, nel filone classico - Medea (2004), Alkestis (2007), Verso Medea (2012-2014), Odissea A/R (2016) ed Eracle (2018) - e in quello che riscrive le fiabe attraverso Le principesse di Emma (Dante, 2014). 
ziale, per essere tradotto scenicamente (secondo lo stile dantiano) con modalità anticonvenzionali, provocatorie e insieme visionarie. Perciò con questo spettacolo subentra ai luoghi deputati stretti (anche perché costretti), chiaroscurali e connotati da costumi e oggetti poveri ma plurivoci - come nella Trilogia della famiglia siciliana (mPalermu 2001, Carnezzeria 2002, Vita mia 2004) - una location performativa più larga, spesso estesa all'intero palco, articolata per moduli scenici che implicano un dinamismo complesso anche dal punto di vista coreografico, e l'accensione dei colori. D'altra parte, le due diverse spazialità sceniche seguitano a coesistere nella produzione teatrale della Dante - pensiamo per la prima alla Trilogia degli occhiali (2011) o a La Scortecata (2017) - conservando entrambe l'obiettivo irrinunciabile di un rapporto vivo e diretto con il pubblico.

\section{AUTORITRATTI SICILIA-MONDO}

Prima di addentrarci nell'esegesi dello spettacolo, allarghiamo lo sguardo sulla produzione dantiana nel suo insieme, con particolare riferimento a quella precedente i Cani, riassumendone aspetti che fanno capo alla sua "autoanalitica" e sempre metaforica sicilianità. Infatti, come già mostrato altrove (Barsotti, 2009), il teatro di Emma Dante e delle sue "famiglie attoriche" riflette ancora, paradossalmente, nel mondo di oggi, l' "effetto Sicilia" colto nel percorso di autori isolani otto-novecenteschi (Verga, Capuana, De Roberto, Pirandello, Lampedusa, Sciascia, Camilleri) $)^{5}$; evocatori di un "altrove" contaminato con il fuori, ma ambiguamente radicato nella propria "sicilitudine", perciò campo d'indagine anche di attori-autori contemporanei (Franco Scaldati, Spiro Scimone, Davide Enia...) che d'altronde, sul proprio corpo, sulle proprie corde

${ }^{5}$ Cfr. Madrignani, 2007. Nel nostro libro abbiamo aggiunto e integrato, da un punto di vista personale, Rosso di San Secondo e, sul versante più avanguardistico del Novecento, Ruggero Vasari, Antonio Aniante, Beniamino Joppolo; anche per quanto riguarda le generazioni contemporanee, la complessità del discorso esige il rinvio all'ampio e articolato quadro tracciato nella Premessa. Il caso Emma Dante. Paradossi siciliani e nel capitolo Sicilia, Palermo, metafore (Barsotti, 2009, pp. 9-39 e pp. 43-50). 
vocali tentano (in vari modi) l' "innesto", trasformano l'Isola in metafora del Mondo. Immagini e messaggi, Leitmotive testimoniano della «perturbante vitalità» dell'Isola, continuando a rappresentare il «dramma strutturale» di una «difficile modernizzazione» (Madrignani, 2007, pp. 16-17), e trascorrendo dal XIX al XX secolo, fino a raggiungere questo secondo millennio.

A partire dalla crisi di trapasso post-unitaria, gli autori siciliani sembrano spinti a ritrarre un mondo deformato da una «filosofia della violenza» così assorbita dal quotidiano e «pervasiva» da apparire un dato «naturale» (Ibidem, 2007, pp. 16-17). Ne deriva l'ambivalenza dell'“autoritratto" - fatto cioè da artisti nativi - d'una terra dove problemi nuovi si innestano su antiche perversioni sociali: maschilismo e servitù femminile (con l'eccezione dell'ambivalente sacralità materna), cattivo ordinamento famigliare riversato nella società fino alle sue propaggini mafiose. Eppure lo stesso autoritratto appare intriso da una passione, talvolta spietata, per la verità ambientale e di conseguenza da "un'arte della responsabilità" e della consapevolezza. È importante notare, d'altra parte, come i nomi degli autori citati siano tutti maschili.

Anche i temi del teatro della Dante ruotano attorno al motivo centrale della "verità", ma ormai a partire dal fisiologico disagio di rapporti familiari de-capitati (in mPalermu non ci sono una madre e un padre comuni, in Carnezzeria ci sono soltanto fratelli...); o dal motivo pirandellianamente insorto del relativismo gnoseologico, ma da un punto di vista diverso, magari attraverso l'ossimoro del Sacro parodiato, nella maschera e non nel volto, come avviene appunto in Cani di bancata. In quest'opera, infatti, la verità sulla «grande famiglia mafiosa» è mascherata dal linguaggio liturgico, dalla sua manipolazione attraverso oggetti legati alla sfera sacrale (santini, candele) in un continuo «rovesciamento di piani dal concreto all'immaginazione, per poi tornare a raccontare il concreto» (Dalisi, 2009, p. 30). Il processo visionario si fonda su una concreta e corposa quotidianità.

«Sacro è tutto ciò che serve per evocare qualcosa», dichiara la Dante, e tuttavia si chiede: «perché devo usare degli oggetti legati alla religione? (...) Perché ho questo (...) immaginario di pungoli comici, quasi da teatro popolare? Il matrimonio, la famiglia, il crocifisso...» (Porcheddu, 
Bologna, 2006, p. 37); potremmo aggiungere l'abito bianco da sposa. La manipolazione per mettere in scena i congegni dell'associazione criminosa investe preghiere e comandamenti, resi funzionali a «evidenziare la matrice sostanzialmente religiosa - nella fattispecie barbarica, pagana - dell'adesione all'organizzazione mafiosa» (Palazzi, 2006). Questa donna di oggi si pone nel solco d'una tradizione in cui il pensiero dominante della Morte è più antico del cristianesimo, innestando concezioni e regole morali della Chiesa su un terreno più oscuro e mitico, che riguarda gli avi greci e mediterranei. Perciò una problematica religiosa in senso lato emerge anche in opere apparentemente laiche; una memoria arcaica, perfino ancestrale, nutre e sostanzia la sua "attuale" poetica del Sacro: «paradossalmente, [un mio] spettacolo diventa più moderno quando io uso il crocifisso (...)!» (Porcheddu, Bologna, 2006, p. 41).

Dei suoi spettacoli si può dare un'interpretazione antropologica oltre che sociale, civile, ed esistenziale; il suo teatro, perché possa parlare ai contemporanei, deve rendere consapevole lo spettatore della durezza e dell'ingiustizia del vivere, ma "senza rassegnazione": dal percorso sulla famiglia siciliana dove mPalermu, Carnezzeria, Vita mia terminano con la morte, a Cani di bancata, sulla famiglia non più come nucleo sociale ma come associazione a delinquere, su cui aleggia alla fine la figura dell'impiccato. Quel senso di "impotenza feroce", che sigilla gli spettacoli della Dante, esprime sia la critica spietata delle regole (soprattutto) non scritte della società sia l'impossibilità, da parte delle creature umane che privilegia (diversi, diseredati, incoscienti), di opporvisi. Ma appunto i suoi "autoritratti" sociali e civili non producono effetti di rassegnazione, bensì indignazione e rabbia, al di là dell'esito spesso desolante e luttuoso.

La ricerca di verità dell'artista palermitana è condotta attraverso un teatro e una Compagnia capace di svelare, nel tempo/spazio di uno spettacolo, passioni e fatti, segreti e bugie, traumi rimossi. «Cerco di sollevare un velo», lei dichiara, perché «sollevare quel velo vuol dire (...) non perdere la memoria, non archiviare le denunce, mostrar la polvere che c'è su tutto...»; e a proposito dei suoi spettacoli: «l'unica regola che abbiamo è "Deve sembrare vero!"» (Meldolesi, Guccini, 2003, p. 21). Ma nella stessa "ricerca vera" della Dante la prospettiva antropologica 
si mescola con interrogativi che rilevano e travestono un'esigenza di autoanalisi; faccio teatro, dice, «per saperne di più, per cercare di capire cosa può diventare questa vita, cosa posso diventare io» (Porcheddu, Bologna, 2006, p. 58).

L'osservazione selvaggia, perseguita da questa "donna di teatro" del Duemila, manifesta un orientamento originale nella riproposta di figure e temi mascherati o aggiornati, sebbene scaturiti da un immaginario comune e da costumi atavici; nella ricerca di motivi e stilemi che ne consentano non l'attualizzazione, ma una resa universalmente umana, ovvero teatralmente contemporanea. I suoi temi sono riconoscibili in un ricorrente rapporto fra vita, morte e sesso, e fra le generazioni, nella demistificazione del Potere e nella valorizzazione del "diverso" - per genere, femminilità compresa, per status sociale - e nella variegata riproposta di una difficile compagine familiare che si rispecchia, paradossalmente, nella stessa gestione della compagnia, nella prospettiva di una infaticabile e incessante trasformazione. Emma è una notevole formatrice di «individui scenici» (Meldolesi, Guccini, 2003, p. 20), al punto che gli spettacoli recenti non fanno rimpiangere l'avvicendamento di quelli del "nocciolo duro" della Sud Costa Occidentale coi più giovani: forse perché fra i maestri della Scuola dei mestieri dello spettacolo del Teatro Biondo di Palermo (da lei fondata nel 2014) non mancano i primi adepti, come Sabino Civilleri e Manuela Lo Sicco. D'altra parte, gli attori "storici" concordano nel ricordare l'«urgenza» esistenziale di quella donna-valanga, per cui tutto era «una questione di vita o di morte», come se dovesse comunicare la «paura di non arrivare a domani» - racconta Sabino Civilleri - «"Domani moriamo, quindi tutto quello che puoi fare devi farlo ora" ci diceva. Questo fu l'inizio» (Bologna, 2006a, pp. 178-179). Ma è e continua ad essere fondamentale che questo "gioco", massacrante ed esaltante, si faccia in gruppo: training, azioni fisiche ed esercizi vocali, variamente attinti, mescolati e ricreati, da Stanislavskij, Artaud, la Malina e la Bausch, Kantor, Grotowski, Barba fino a Vacis. Input e condensazione finale appartengono alla Dante, nel mezzo agiscono i contributi individuali e collettivi dei suoi attoricoautori, in alcuni dei quali si rispecchia o si ricerca; come in Manuela Lo Sicco che ha rappresentato una sorta di autoproiezione femminile, 
da attrice-autrice a medium privilegiata di quella donna un po' pazza ma esperta che afferma: «In realtà, io non sono una regista vera» (Porcheddu, Bologna, 2006, p. 41).

La sua istanza drammaturgica e performativa è strutturalmente nonlineare, difficilmente sintetizzabile, non con-causale ma analogica. Già su questo piano, in mPalermu, si svolge il racconto paratattico d'una giornata particolare ed emblematica, dal "risveglio" d'una famiglia eterogenea e senza padre al "grande sonno" che l'arresta sulla soglia d'una tentata evasione (dalla casa, dalla Città, dall'Isola, dal boccascena trasparente ma frontale al pubblico); o la resa d'un episodio cruciale, in Carnezzeria, d'altra famiglia senza padre e senza madre, tre fratelli e una sorella sfantasiata e incinta, trasmigrati allo scopo di recitare per quest'ultima, in una scena-piazza, un grottesco matrimonio riparatore che servirà al suo abbandono e condurrà al suo suicidio "alla rovescia". In questo contesto la Lo Sicco, timida-aggressiva Rosalia in mPalermu, Nina vergine madre e puttana in Carnezzeria, si scompiglia i capelli, con un gesto da Erinni che prepara la sua Mammasantissima bestiale e gelida di Cani di bancata.

\section{DEL FEMMINILE E DELLE MAFIE: \\ IL CASO CANI DI BANCATA}

In merito al discorso donna-teatro-mafie, parto dal titolo "editoriale" di un'intervista di Rodolfo Di Giammarco contemporanea al debutto di Cani di bancata: Emma Dante: Io, siciliana vi dico che la Mafia è don$n a$. Titolo che fraintende clamorosamente il senso e la lettera stessa delle parole dell'autrice: «Premetto che sono siciliana, che vivo a Palermo, e che so distinguere l'atteggiamento mafioso (un bagaglio espressivo fatto di pregiudizi, sospetti e omertà) dalla mafia in quanto tale. Ma so anche che ogni atteggiamento favorisce l'attecchire di certi meccanismi. Basta un niente per essere infettati» (Di Giammarco, 2006). Quanto alla Mammasantissima al femminile spiega: «Perché la mafia s'incontra in un luogo chiamato 'casa santa', e una donna, una vaiassa [dal napoletano] sgraziata incarna idealmente la madre di tutti loro (lo strascico di lei sarà la tovaglia per un rito eucaristico collettivo, n. d. r.), li nutre, li 
fa studiare, li fa infiltrare nella società applicando un'iconografia cattolica, di cui ho riprodotto [manipolandoli] dieci comandamenti funesti» (Ibidem). E in un'altra intervista dello stesso anno la Dante polemizza proprio con l'interpretazione che identifica donne e mafia: «Tutti dicono di sapere tutto sulla mafia e per questo Cani di bancata è urtante (...). Ma lo sapete che la donna è bandita dalle tavolate in cui si decidono le sorti di qualcuno? E che quindi mettere una donna a capo di una cosca è il simbolo di qualcos'altro?». E aggiunge: «è importante l'inserimento della figura femminile che muove i fili di queste pedine. Non è un boss, ma incarna la Mafia, altrimenti avrei rifatto il Padrino. Ma io volevo suscitare un sentimento di schifo, mentre chi non è stato affascinato dal personaggio di Marlon Brando?» (Polidoro, 2006).

Di rovesciamento in rovesciamento - che è poi la cifra stilistica della Dante - mettere una donna a capo d'un mondo mafioso implicante 'ndragheta e camorra, oltre a costituire un «avvenimento», ossia un «passaggio del limite» (Lotman, 1972, p. 271), una sorpresa all'epoca del debutto, da un lato trae linfa critica e vitale dalla tradizione e dal costume isolano, dall'altro, infrangendo un tabù, persegue lo scopo di rendere tanto più "schifosa" la simbolizzazione carnale della Mafia.

Dal punto di vista dell'intreccio dell'opera, il tessuto drammaturgico nella versione finale è composto da quattro sequenze (non scandite nello spettacolo ma nel testo poi pubblicato): "Prologo", "Rito di affiliazione", "Il rito del pane e del vino", "La rivelazione". «Più che raccontare una trama definita, inscena[no] una specie di lungo rito di iniziazione e di appartenenza, un inquietante cerimoniale di fedeltà e sottomissione», secondo Palazzi (2006); eppure anche altro... Il filo rosso ma sarebbe meglio dire grigio (data la divisa che indossa) - è costituito dall'avventura di un estraneo, il capostazione Liborio Pagnino (Stefano Miglio) costretto ad assistere all'affiliazione d'un nuovo adepto, don Sasà detto lo Spagnuolo (Salvatore D'Onofrio), cui egli è debitore d'un posto di lavoro ottenuto con l'espediente d'una falsa invalidità. Gli occhiali inforcati lo fanno "non vedere", perciò è responsabile dell'incidente letale occorso a un collega, ma coperto dall'omertà. Da parte sua Liborio deve tacere su quanto vedrà, e ripetere la formula del segno della croce così adattata: «Nel nome del padre, del figlio, della madre, 
dello spirito santo, amen» (Dante, 2007a, p. 103) $)^{6}$. L'iniziazione di don Sasà mescola - come anticipato - segni rituali religiosi e propriamente mafiosi. Egli al principio è come morto, e quando resuscita dovrà affrontare due prove (il bacio e il pestaggio) cui lo sottopone Mammasantissima/Lo Sicco, la creatura oscuramente vestita da maschio che, con movenze mimiche e gestuali cagnesche, si spoglierà svelando un abito femminile nero, per poi indossare un enorme - e abnorme - abito bianco da sposa. Infatti, dopo l'accoglimento del neofita, si rappresenta 1'“ultima cena" d'una infernale e bestiale famiglia, schierata ai lati d'un desco al cui vertice, frontalmente, siederà Madre Mafia, con il lungo strascico come copritavolo. Il quadro si sommuove dal momento in cui la capobranco sacrale, dopo avere chiamato Liborio alla sua destra (perché è un "uomo perbene"), sconvolge l'intera scala gerarchica dei posti, scatenando rimostranze reciproche tra gli affiliati, che culminano in una grande zuffa. Ne resta vittima l'estraneo, sin dall'inizio a disagio, e infine violentemente pestato e costretto dagli accoliti dell' "onorata società" a confessare pubblicamente il proprio reato: a causa degli occhiali che gli oscurano la vista, il Cst ha fatto partire il treno mentre un ferroviere faceva controlli sotto il convoglio, cosicché la testa del malcapitato è stata tranciata di netto. Le relative azioni di bilanciato disordine rispondono non solo a una esattezza coreografica di straordinario impatto, ma anche a quel processo di "musicalizzazione" (secondo lo stilema dantiano del suono agito e non decorativo) che fa assumere a scene collettive forti e semanticamente brutali un effetto tragi-comico: quando scoppia il caos fra i malavitosi, gli attori corrono forsennatamente da un lato all'altro del palco, sputandosi addosso e spingendosi a vicenda, al ritmo dei Jethro Tull; mentre perseguitano Liborio fino al parossismo, accerchiandolo e lanciandone ripetutamente in aria il cappello (che simula la testa mozzata), il ritmo è scandito dalla musica di All about me, ad altissimo volume.

6 La formula liturgica manipolata, Leitmotiv del dramma, tornerà nella versione originaria alla fine dello spettacolo, quando il senso della recita si legherà in modo blasfemo al disegno della sua finta sparizione concepito dalla Madre-Mafia. 
Nell'epilogo la Madre Mafia, che fino a quel momento ha guidato e nutrito il clan con la ferocia e col sangue, spiega il suo nuovo piano, chiamando a raccolta i figli con i nomi e i ruoli che svolgono nella società parallela, così rivelandoli al pubblico. Lei cesserà di esistere e loro asseconderanno la sua finzione, trasformando in normalità la violenza; divenuta invisibile, garantisce la continuità dell'associazione criminale. Con l'ultimo travestimento di Mammasantissima, di nero velata, che passa di soppiatto dietro alla schiera degli attori e marchia la schiena di ciascuno con una sillaba fino a formare la frase "Io-ma-dre-vi-af-fi-dol'Ita-li-a", si chiude la sua azione diretta; non senza avere provveduto all'impiccagione dell'estraneo, testimone da sopprimere.

Sappiamo che il titolo dell'opera riprende l'espressione palermitana con cui sono indicati i cani che racimolano avanzi e scarti insinuandosi sotto i banchi del mercato. In quei cani si metaforizzano i malavitosi: non a caso come animali si azzuffano, sotto gli occhi e la regia della Cagna madre dall'alto del suo scranno. Ma soprattutto la Lo Sicco è "cerbero" infernale: durante il «rito di affiliazione», rimane sul proscenio, a quattro zampe, ringhiando, spargendosi il rossetto sul viso e sbavando; durante il «rito del pane e del vino» divora il pasto e lo sputa sulla tovaglia. Lo Sicco/Mammasantissima accentua in questi passaggi le sue caratteristiche recitative, deformandole: la mimica «discreta» (Molinari, Ottolenghi, 1977, p. 108) diventa quasi fissa, una maschera dalla bocca storta, occhi spalancati, voce ancora più innaturale e gesti più aperti, aggressivi e possessivi, incombendo costantemente anche sullo spettatore ${ }^{7}$.

Eppure, la genesi della protagonista rimane visionaria, secondo la prospettiva dantiana: «Quello sguardo di Manuela che diventa lo sguardo di qualcun altro... Questo è il fantasmino (...). Io, qualche volta, quando facevo l'attrice avevo sfiorato questa "trasformazione": ma l'ho scoperta nei miei attori» (Porcheddu, Bologna, 2006, p. 39). E, da parte

7 Rispetto alla fase laboratoriale, il gruppo femminile delle «Menadi, le detentrici di crudeltà e ferocia, le portatrici di violenza» (Bologna, 2006b, p. 159), si concentra nella figura-simbolo di Mammasantissima e l'idea iniziale di uno sguardo straniero sulla comunità è conservata nel personaggio di Liborio Paglino. Cfr. anche Dalisi, 2007, pp. 108-109. 
sua, anche la Lo Sicco per trasformarsi in Mammasantissima ricorre a un doppio sguardo; durante le prove ha guardato, dall'alto del trono dov'era stata apparentemente abbandonata, Emma «seduta per terra» a lavorare con i «cani»: quindi «io vedevo lei e loro ed ho studiato proprio (...) come guardava gli altri mentre facevano le azioni e come li doveva portare per farli muovere (...). Il corpo è nato da un'improvvisazione (...), la respirazione (...), la schifezza animale, la bava». Ma «l'anima» della capomafia «non deriva solo dal suo corpo (...) perché mammasantissima ha una testa e un corpo» (Galletti, 2009, p. 85). Un rispecchiamento, al femminile, angoscioso e perturbante.

Nonostante riprese e autocitazioni tematiche e stilistiche (vestizioni, svestizioni, scambio d'abiti e di generi, giochi di luci, suoni e soprattutto di corpi) lo spettacolo - come anticipato - si presenta anomalo rispetto ai precedenti, sotto diversi punti di vista. Già per il numero di attori coinvolti: prima non erano più di quattro o cinque; qui sono invece undici, compresa la Madre Mafia. I dieci figli di quella Madre terribile e prevaricante riempiono il palco, senza lasciare spazio a chi se ne vorrebbe allontanare; così come riempiono il nostro spazio vitale, senza che ce ne accorgiamo: ognuno di loro - scopriamo alla fine - occupa un ruolo sociale o politico o religioso importante nel paese. Anche il disegno scenico è nuovo, pur richiamando (per la stessa numerosità degli attori) un altro spettacolo anomalo, la prima Medea del 2004. La scena è essenziale ma complessa, basata su una struttura modulare praticabile e polisemica: nel primo quadro sedie dall'altissimo schienale funzionano come una specie di rastrelliera di Pupi, dietro la quale gli attori si vestono, incastonati, ma l'impalcatura potrebbe simulare anche l'ipotetico ponte di Messina (su cui alcuni camminano) o la facciata di una cattedrale; le stesse sedie diventeranno poi, degradando verso il boccascena, posti della tavolata e, successivamente, ali di un tribunale-parlamento.

Inoltre, qui la Dante non usa mezze misure: condanna assolutamente la Mafia, a partire dal titolo della pièce. Come già notato, gli attori/cani assumono spesso movenze zoomorfe, quando non marciano in "schiera" avanti/indietro senza sosta, sperimentando collettivamente, per la prima volta, quella camminata da "picciotto" che ne millanta la mascolinità (mani in tasca, bacino proteso, sorriso strafottente e cappel- 
lo sul viso). Mascolinità inficiata qui - come in altri casi - dagli stessi cappelli colorati da donna ${ }^{8}$. Ad ogni modo, questo spettacolo è diverso proprio perché addita inequivocabilmente un colpevole; laddove invece, nei precedenti, perfino i personaggi più efferati (come i fratelli di Carnezzeria), non sono giudicati colpevoli del tutto, o perché inconsapevoli, o perché a loro volta vittime.

Da una parte, è inevitabile che dopo avere messo in scena il microcosmo della famiglia siciliana, racchiudendovi abusi, tensioni, regole, cerimonie, infrazioni "piuttosto estreme", l'autrice abbia sentito il bisogno di affrontare il tema della macrofamiglia mafiosa in Cani di bancata, cercando di capire quali possano essere i meccanismi umani che hanno originato e alimentano il fenomeno. È un mondo che appartiene alle sue radici, essendo siciliana, non può non sottoporlo a indagine. Non è un caso, d'altronde, che per scrutare quel mondo si sia ispirata dal punto di vista letterario - oltre che a Il Grande Inquisitore, capitolo di I fratelli Karamàzov di Dostoevskij - ai romanzi di Sciascia, da A ciascuno il suo (1966) a Todo modo (1976), passando comunque per Il contesto, magari attraverso la visione di Cadaveri eccellenti (1976) di Francesco Rosi.

Leonardo Sciascia, mosso da passione lucida per la verità ambientale e di conseguenza ancora più consapevole di professare ed esercitare un'arte della responsabilità e della coscienza - dopo avere acutamente diagnosticato il "complesso di superiorità siciliano", individuandone alcune cause storiche - a partire da Il contesto (1971) finisce quasi, paradossalmente, per ratificarlo nella pessimistica chiusura del romanzo:

Un paese immaginario [...]. E si può anche pensare all'Italia, si può anche pensare alla Sicilia [...]. Possono essere siciliani e italiani [...] gli accidenti, i dettagli; ma la sostanza (se c'è) vuole essere quella di un apologo sul potere nel mondo, sul potere che sempre più degrada nella impenetrabile for-

8 Già in $m$ Palermu, Giammarco/Civilleri si pavoneggia muovendo «ritmicamente le spalle ed il bacino, col petto in fuori come un picciotto» (Barsotti, 2009, p. 160). Patrizia Bologna cita a riguardo Mario Perniola, cogliendo una particolare carica espressiva in relazione all'abbigliamento: «Un cappello femminile usato su un corpo che cammina come se stesse scopando dà un'idea di maschio e femmina insieme, di eros ambivalente» (Bologna, 2006, p. 157). 
ma di una concatenazione che approssimativamente possiamo dire mafiosa.

(p. 122)

Se è vero che la realtà nazionale, e non solo quella, presenta incastri perturbanti con i giochi di Potere rilevati da Sciascia ("mafiopoli"), l'angolazione della Dante ripropone nel suo Cani di bancata l'ottica siciliana, attraverso quella lente d'ingrandimento speciale che continua a focalizzare i mali dell'Isola, ma li dilata sino a farne una terrificante metafora del Mondo. Tuttavia, questo lavoro (molto discusso dalla critica) sembra mostrare una rivalsa radicale, mediante due provocatori "rovesciamenti". Alla fine, lo spettatore è messo di fronte a una gigantesca cartografia dell'Italia spezzata e capovolta, dove la Sicilia invece che al fondo si trova in cima allo stivale; anziché essere un'appendice coloniale domina con il suo invisibile sistema di potere l'intero continente. Resta, nel rovesciamento di genere del ruolo di capo branco, un senso latente ma complesso d'ambiguità; non va frainteso (ha ragione l'artista) secondo una banale e maschilista prospettiva. Quel rovesciamento implica radici, il cui intreccio è e dev'essere difficile districare: affondano nell'humus atavica d'una terra socialmente, economicamente e politicamente dominata dal maschio, eppure famigliarmente affidata alla guida sacrale della donna-Madre. Perciò la Mafia (con la camorra, la 'ndrangheta) è metaforizzata all'inizio in una "casa santa", nel contesto d'una famiglia numerosa ma chiusa, in cui si entra ma da cui non si esce altro che con la morte.

Il nucleo è riconducibile, in dimensioni ridotte e più letterali, alla donna-Madre di Vita mia - nella quale rivive l'archetipo della Famiglia intesa nel senso d'una forma mentis siciliana, ma sul versante matriarcale, non su quello patriarcale - che, da sola, esercita un potere esclusivo e frustrante sui tre figli di cui teme l'uscita per eccesso di protezione. Qui, al contrario, la Madre che esercita il suo dominio sulla più ampia famiglia-Mafia non è connotata in alcun modo da preoccupazioni affettive, ma manifesta soltanto - e schifosamente - intenti legati al Potere, con la P maiuscola. Non solo, ma alla fine finge di scomparire, di non esserci - assecondando il Leitmotiv convenzionale che ne nega l'esistenza -, ordina anzi ai suoi figli di uscire nel "paese del mondo", fingendo 
essi stessi di non essere altro che quel che sembrano (imprenditori, banchieri, politici, giornalisti e alti prelati), così da infiltrarsi meglio, senza apparente violenza, e più proficuamente nella società e infettarla.

Lo scandalo di questo «crudo ed emblematico ritratto della Mafia di oggi, del cerimoniale e delle nuove strategie di un suo vertice» (Di Giammarco, 2006) non è nei contenuti, che secondo la Dante sono noti a tutti; bensì nella forma drammaturgica e spettacolare in cui quei contenuti precipitano brutalmente, perché la metaforizzazione del processo s'incarna in un corpo a corpo con lo spettatore, segnatamente nel finale. Man mano che gli attori/maschi ricevono la "marchiatura" posizionano la "maschera" sulla parte posteriore della testa, mostrano per qualche istante il volto contorto in un'esagerata risata, poi si voltano e, sfilandosi le mutande, si masturbano di fronte alla carta geografica, che intanto si è innalzata, facendo scendere un cappio e una zavorra. L'attrice/Madre lega le mani a Liborio, gli sfila gli occhiali e gli stringe la corda intorno al collo, slegando il contrappeso. Solo tre attori restano in piedi a formare la parola "ma-fi-a" e il buio cala sul testimone impiccato.

Un'ultima nota riguarda l'ambiguità stessa della metaforizzazione Mammasantissima-Mafia, riassumendo la serie di travestimenti osservati, che ne mettono in rilievo una ricorrente androginia (del resto si dice in gergo mafioso "il" mammasantissima). In principio la Lo Sicco è in completo nero da maschio, poi scioglie i capelli e li scuote, si muove come un'orribile cagna e scopre una veste nera da donna, finché non s'infila il candido abito nunziale, e solo allora si pone al vertice della scala di sedie, mentre lo strascico diviene tovaglia per la mensa; infine si riveste e si vela di nero per scomparire, dopo avere scritto in rosso sulle schiene nude dei figli la formula del finto commiato ("Io-ma-dre-vi-affi-do-l'Ita-li-a").

\section{BIBLIOGRAFIA}

Barsotti, A. (2009). La lingua teatrale di Emma Dante. mPalermu, Carnezzeria, Vita mia. Pisa: ETS. 
Barsotti, A. (2010). Emma attraversa lo specchio: postdrammatico VS drammatico. Prove di Drammaturgia, XVI/1, 26-32.

Barsotti, A. (2011, June 11). La Trilogia degli occhiali di Emma Dante. Dal testo alla scena? Drammaturgia.it. Retrieved from http://drammaturgia. fupress.net/saggi/saggio.php?id=5025.

Barsotti, A. (2014). Dalla Sicilia al Nord Europa: la morte in scena al femminile e nel femminile [Emma Dante-Jan Fabre]. In R. Carpani, L. Peja \& L. Aimo (Eds.), Donne Personaggi e Interpreti. Studi per Annamaria Cascetta (pp. 395-404). Milano: Vita e Pensiero.

Barsotti, A. (2017a). Odissea A/R di Emma Dante. Riattivazione del mito in due movimenti. Il Castello di Elsinore, 76, 91-127.

Barsotti, A. (2017b). Le sorelle Macaluso tra la vita e la morte nella tetralogia della famiglia siciliana. Arabeschi, luglio-dicembre, 10. Retrieved from http://www.arabeschi.it/le-sorelle-macaluso-tra-la-vita-e-morte-nellatetralogia-della-famiglia-siciliana- $/$.

Bologna, P. (2006a). Uno spazio dove tutto è possibile. Intervista a Sabino Civilleri. In A. Porcheddu (Ed.), Palermo dentro. Il teatro di Emma Dante (pp. 177-182). Civitella in Val di Chiana (Ar): Zona.

Bologna, P. (2006b). Verso Purgatorio. In A. Porcheddu (Ed.), Palermo dentro. Il teatro di Emma Dante (pp. 142-172). Civitella in Val di Chiana (Ar): Zona.

Dalisi E. (2007). "Il teatro non si può capire, si deve fare". Diario di viaggio verso Cani di bancata. Hystrio, XX/1, 108-109.

Dalisi, L. (2009). Messa in scena della mafia. Cani di bancata: il metodo maieutico di Emma Dante. Napoli: Dante \& Descartes.

Dante, E. (2007a). Cani di bancata. Hystrio, XX/1, 102-107.

Dante, E. (2007b). Carnezzeria. Trilogia della famiglia siciliana. Roma: Fazi.

Dante, E. (2014). Le principesse di Emma. Lavis (TN): Baldini \& Castoldi.

Di Giammarco, R. (2006, October 31). Emma Dante: Io, siciliana vi dico che la Mafia è donna. Intervista a Emma Dante. la Repubblica.

Ficara, M., Pompei, F. (2007). La pratica che insegna ad amare. Intervista ad Emma Dante. In M. Ficara (Ed.), Donne di teatro e culture della (r)esistenza, Percorsi dai dieci anni di "The Open Page” (pp. 189-196). Roma: Editoria \& Spettacolo.

Galletti, E. (2009). Intervista a Manuela Lo Sicco. In Eadem, La figura della donna nel teatro di Emma Dante: Manuela Lo Sicco (Master's thesis) (pp. 72-83). Pisa: Università di Pisa, 
Lotman, Ju. M. (1972) La struttura del testo poetico, a cura di e trad. di E. Bazzarelli. Milano: Mursia.

Madrignani, C.A. (2007). Effetto Sicilia. Genesi del romanzo moderno. Macerata: Quodlibet.

Mariani, L. (2016). Registe di teatro in Italia. Ce n'est qu'un début... Culture Teatrali, 25, 108-123.

Meldolesi, C., \& Guccini, G. (2003). Il teatro di Emma Dante. Appunti sulla ricerca di un metodo. Presentazione. Prove di Drammaturgia, 18, 21-22.

Palazzi, R. (2006, December 3). La mafia senza coppole e gessati. Il Sole 24 ore.

Polidoro, P. (2006, November 27). "Io, le donne e la mafia". Emma Dante: a teatro "mammasantissima" è femmina. Il Messaggero.

Porcheddu, A., \& Bologna, P. (2006). La strada scomoda del teatro, Intervista a Emma Dante. In A. Porcheddu (Ed.), Palermo dentro. Il teatro di Emma Dante (pp. 27-77). Civitella in Val di Chiana (Ar): Zona. Sciascia, L. (1971). Il contesto. Una parodia. Torino: Einaudi.

Riassunto: Il saggio riflette su Emma Dante, artista polivalente (capocomica e attrice-autrice di teatro, attrice e regista di cinema, regista di opere liriche), che costituisce un'eccezione nel panorama italiano in quanto donna di scena e donna di libro. La sua storia condensa esperienze e competenze diverse, apparentemente contrastanti (Accademia d'Arte drammatica a Roma, magistero di Vacis a Torino, laboratori con Cesare Ronconi) prima della fondazione del gruppo Sud Costa Occidentale a Palermo, nel 1999, con le sue successive trasformazioni fino ad oggi. Ne nasce un'anomala figura di "matriarca", nel filone fecondo della nuova drammaturgia siciliana, che dalla terra d'origine trae succhi ma con i quali alimenta un teatro al tempo stesso collettivo e autoriale; drammatico (anzi tragicomico) e postdrammatico, di rispondenza europea, non senza suscitare polemiche e attacchi, essendo teatro scomodo e foriero di rischiose autoanalisi. L'esame del suo spettacolo Cani di bancata (2006) intende mettere in luce temi e stili connessi a un femminile che esula dal gender (in senso stretto) ma capace di farne la metafora di un mondo e di una diversità umana che, attraverso uno sguardo complice e dissacrante, ci coinvolge e ci turba.

Parole chiave: Emma Dante, teatro, Cani di bancata, Sicilia, mondo 